\title{
Template Guided Visual Inspection
}

\author{
A. Noble, V.D. Nguyen, C. Marinos, \\ A.T. Tran, J. Farley, K. Hedengren, J.L. Mundy \\ GE Corporate Research and Development Center \\ P.O. Box 8, 1 River Road Schenectady, NY. 12301. USA.
}

\begin{abstract}
In this paper we describe progress toward the development of an X-ray image analysis system for industrial inspection. Here the goal is to check part dimensions and identify geometric flaws against known tolerance specifications. From an image analysis standpoint this poses challenges to devise robust methods to extract low level features; develop deformable parameterized templates; and perform statistical tolerancing tests for geometry verification. We illustrate aspects of our current system and how knowledge of expected object geometry is used to guide the interpretation of geometry from images.
\end{abstract}

\section{Introduction}

Automatic visual inspection is a major application of machine vision technology. However, it is very difficult to generalize vision system designs across different inspection applications because of the special approaches to illumination, part presentation, and image analysis required to achieve robust performance. As a consequence it is necessary to develop such systems almost from the beginning for each application. The resulting development cost prohibits the application of machine vision to inspection tasks which provide a high economic payback in labor savings, material efficiency or to the detection of critical flaws involving human safety.

The use of Computer Aided Design (CAD) models has been proposed to derive the necessary information to automatically program visual inspection $[16,2]$. The advantage of this approach is that the geometry of the object to be inspected and the tolerances of the geometry can be specified by the CAD model. The model can be used to derive optimum lighting and viewing configurations as well as provide context for the application of image analysis processes.

On the other hand, the CAD approach has not yet been broadly successful because images result from complex physical phenomena, such as specular reflection and mutual illumination. A more significant problem limiting the use of CAD models is that the actual manufactured parts may differ significantly from the idealized model. During product development a part design can change rapidly to accommodate the realities of manufacturing processes and the original CAD representation can quickly become obsolete. Finally, for curved objects, the derivation of tolerance offset surfaces is quite complex and requires the solution of high degree polynomial equations [3].

An alternative to $C A D$ models is to use an actual copy of the part itself as a reference. The immediate objection is that the specific part may not represent the ideal dimensious or other properties and without any structure it is impossible to know what attributes of the part are significant. Although the part reference approach has proven highly successful in the case of VLSI photolithographic mask inspection $[5,14]$ it is difficult to see how to extend this simple approach to the inspection of more complex, three dimensional, manufactured parts without introducing some structure defining various regions and boundaries of the part geometry. The major problem is the interpretation of differences 
between the reference part and the part to be inspected. These differences can arise from irrelevant variations in intensity caused by illumination, uniformity or shadows. Even if the image acquisition process can be controlled, there will be unavoidable part-to-part variations which naturally arise from the manufacturing process itself, but are irrelevant to the quality of the part.

In the system to be described here we combine the best features of the CAD model and part reference approaches by introducing a deformable template which is used to automatically acquire the significant attributes of the nominal part by adapting to a large number of parts (e.g. 100). The template provides a number of important functions:

- Part feature reference coordinates

- Feature tolerances for defining flaw conditions

- Domains for the application of specialized image feature extraction algorithms.

In Section 2 we consider the theoretical concepts which determine the general structure of a constraint template. Section 3 describes the general design and principal algorithms used in the current prototype inspection system. Experimental results demonstrating constraint templates applied to X-ray images of industrial parts are given in Section 4. We conclude in Section 5.

\section{Constraint Templates}

We have based the design of our inspection system on the definition of a template which consists of a set of geometric relationships which are expected to be maintained by any correctly manufactured instance of a specific part. It is important to emphasize that the template is a generic specification of the entire class of correct instances which can span a wide range of specific geometric configurations. We accommodate these variations by solving each time for an instance of the template which satisfies all of the specified constraints, while at the same time accommodating for the observed image features which define the actual part geometry. Currently, the system is focused on single 2D views of a part, such as X-ray projections. However, there is no limitation of the general concept to a single image, so that multiple $2 \mathrm{D}$ views or $3 \mathrm{D}$ volume data could be interpreted by a similar approach.

More specifically, the template is defined in terms of the following primitive geometric entities; point, conic (ellipse, circle, hyperbola,line), bezier curve. These primitive curve types can be topologically bounded by either one or two endpoints to define a ray or curve segment. The geometric primitives are placed in the template in the context of a set of geometric relationships. The set of geometric constraints available are as follows:

Incident Two geometric entities have at least one point in common.

Coincident Two entities have exactly the same descriptive parameters. For example, two points are at the same location.

Location Two points are constrained to be a fixed distance apart. Or more generally, the position of two entities is constrained by a distance relation.

Angle The relative orientation of two entities is fixed.

Parallel A specific case of angle, i.e. $0^{\circ}$.

Perpendicular A specific case of angle, i.e. $90^{\circ}$.

Symmetry Symmetry can be defined with respect to a point or a line. That is a reflection across the point or line leaves the geometric figure unchanged.

Tangent Continuity Two curve primitives are constrained to have equal tangents at a point. Equal Size The size of a primitive is defined more formally below but is essentially the scale of the entity. This constraint maintains two primitives to have equal scale factors. 
Size in Ratio Two primitives have some fixed ratio in scale factor.

Linear Size A set of entities are related by a linearly varying scale factor. This relationship is often observed in machine parts.

Linear Spacing The distance between a set of entities varys linearly over the set. Again, this constraint is motivated by typical part geometries.

\subsection{The Configuration Concept}

We have developed the concept of the configuration which provides a systematic approach to the symbolic definition of geometric entities and many of the geometric relationships just defined [12]. The geometric constraints are ultimately represented by a system of polynomials in the primitive shape variables and the constraint parameters.

Except for scalar measures such as length and cosine, all geonetric entities are represented by configurations, which have parameters for the location, orientation, and size of the primitive shapes. Symbolically, these slots are represented by $2 \mathrm{D}$ vectors of variables. The location of a shape is described by $\mathbf{l}^{T}=\left(l_{x}, l_{y}\right)$. This location is usually the center or the origin of the local frame of the primitive shape. The orientation of a shape in the plane is described by an angle $\theta$, or by a unit vector $\mathbf{o}^{T}=\left(o_{x}, o_{y}\right)=(\cos \theta, \sin \theta)$. The later is used to avoid trigonometric functions and to use only polynomial functions of integer powers. The size of a shape, like for an ellipse, is represented by a vector having 2 scale factors, $\left(k_{x}, k_{y}\right)$ along the major and minor axes. To avoid division of polynomials, the inverse of the size is represented: for example, $\mathbf{k}^{T}=\left(k_{x}, k_{y}\right)=\left(a^{-1}, b^{-1}\right)$ for an ellipse.

The configuration is an affine transformation matrix representing the translation, rotation, and scaling from the local coordinate frame $(X, Y)$ of the shape to the image frame $(x, y)$ :

$$
\left(\begin{array}{l}
X \\
Y
\end{array}\right)=\left(\begin{array}{cc}
k_{x} & 0 \\
0 & k_{y}
\end{array}\right)\left(\begin{array}{cc}
\cos \theta & \sin \theta \\
-\sin \theta & \cos \theta
\end{array}\right)\left(\begin{array}{l}
x-l_{x} \\
y-l_{y}
\end{array}\right)
$$

\section{System Design}

\subsection{Philosophy}

The inspection system operates in one of two functional modes; inspection template acquisition mode or part inspection mode, Figure 1. Inspection template acquisition, involves the derivation of a constraint template which encapsulates the expected geometry of a "good" part. Initially a template is created manually by a user through a graphical interface with the aid of blueprint specifications or inspection plans. Once a template is created, the system is run on a suite of images of "good" parts to refine the nominal template parameters and provide statistical bounds on parameter values. The end result is a template description which includes correction for inaccurate placement of primitives in the initial template creation process and which accurately reflects the true part geometry.

Part inspection involves making decisions about whether parts contain defects. For example, parts must not contain flaws produced by poor drilling and part dimensions must satisfy geometric tolerance specifications. In terms of image analysis tasks this process involves first extracting empirical geometric features from the image data via image segmentation and local feature parameterization. Global context for decision-making is provided via the inspection template which is deformed to the empirical primitives by first registering the template to image features and then applying nonlinear optimization techniques to produce the "best-fit" of the template to the empirical features. Finally, the deformed template description is used for verification of part feature dimensions and to provide the context for the application of specialized algorithms for characterizing local flaws. 


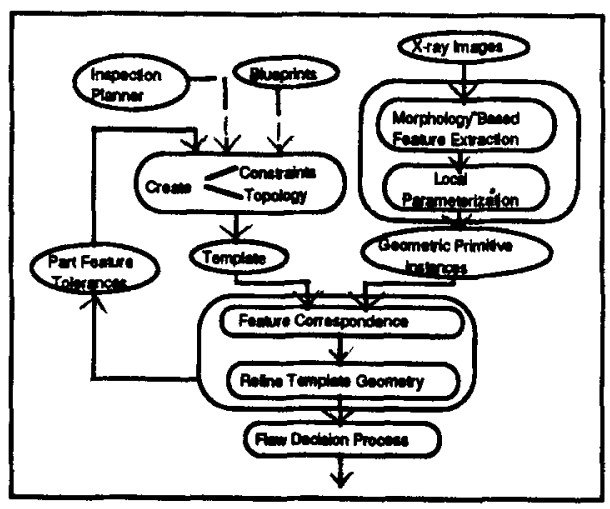

Fig. 1. Flowchart of critical components of the inspection system.

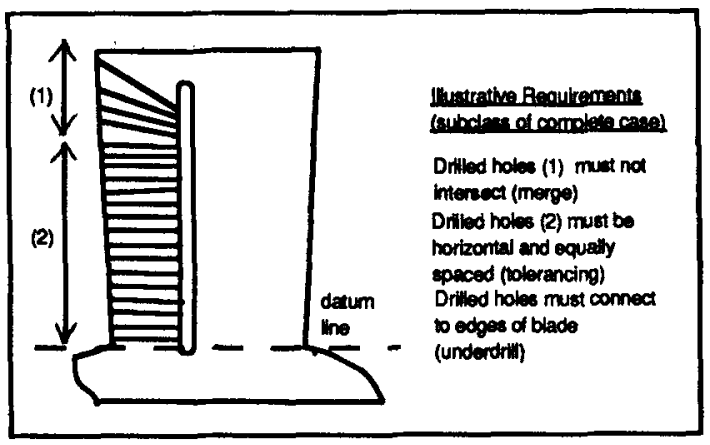

(a) (b)

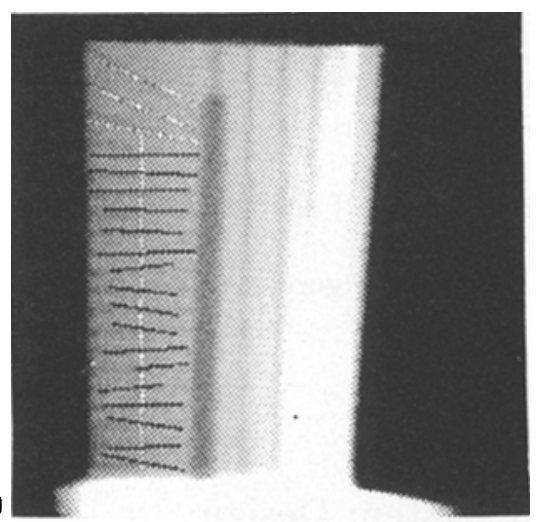

Fig. 2. (a) A simplied example of requirements for an inspection template, and; (b) A snapshot in the process of template construction illustrating the introduction of a constraint after line primitive creation.

\subsection{System Components}

Essentially the inspection system can be divided into four functional modules: template creation; image feature extraction; template refinement and flaw decision-making.

Template Creation. A simplified example to illustrate the requirements for an inspection template is shown in Figure $2 a$. The general template creation process involves first specifying a set of geometric primitives and then establishing the relationships between them. In our system this is achieved using a graphical template editing tool which allows the user to build a template composed of a selection of the 4 types of geometric primitive specified in section 2 which are related by any of 12 possible constraint types. Figure2b illustrates a "snap-shot" view in creating a template.

Image Segmentation. The extraction of geometric primitives is achieved using a morphology based region boundary segmentation technique. Details of this algorithm can be found elsewhere [13]. This algorithm locates, to pixel accuracy, boundary points on either side of an edge as half boundaries which are 4-connected pixel chains. A typical 
output from the algorithm is shown in Figure $3 \mathrm{~b}$ where both edges of the regions are highlighted.

To detect subtle changes in image geometry and to achieve accurate feature parameterization we have implemented a subpixel residual crossing localization algorithm. A morphological residual crossing is defined as the zero-crossing of the signed max dilationerosion residue, $f_{\operatorname{maxder}}(f)[13]$ :

$$
\left|f_{\text {maxder }}(f)\right|=\max \left[\left|f_{e r}(f)\right|,\left|f_{d r}(f)\right|\right]
$$

where, $f_{e r}(f)=f-f \ominus B, f_{d r}(f)=f \oplus B-f, f$ is an image, $B$ a structuring set, $f \oplus B$ and $f \ominus B$ are dilation and erosion respectively, and the sign of the residual satisfying the magnitude condition is attached to $f_{\text {maxder }}$. Subpixel residual zero-crossings are found using the following algorithm:

1. First, the residual values are interpolated by a factor 2 using a $7 \times 7$ pixel separable cubic spline interpolation filter [10]. This is done in the neighborhood of each point which belongs to a pixel accurate region boundary contour.

2. Then, the residual crossing locations are located from the interpolated max dilationerosion residue responses. This is achieved using a modified version of the predicatebased algorithm proposed by Medioni and Huertas for locating zero-crossings of the Laplacian of the Gaussian operator[8]. The result is an 8-connected single-pixel wide edge map with boundaries located to 0.5 pixel accuracy, Figure 3c.

In the application, drilled hole features appear as dark elongated intensity regions in an image, of width approximately 4 pixels. They are extracted using the following algorithm. First, the image is enhanced by applying a morphological closing residue operator [9], using a disk of radius 5 pixels. Then, region boundary segmentation is applied to the filtered image. Figure $3 \mathrm{~b}$ shows the closed contours detected to pixel accuracy where both sides of the edge between the two regions are highlighted. Subpixel precision edge locations are shown in Figure 3c.

Empirical Feature Construction and Feature Correspondence. The objective here is to derive a geometric representation from the image data which can be associated with the constraint template primitives. We view the features which are extracted by image segmentation to be empirical versions of the ideal template primitives.

In the current implementation, correspondence is carried out as a search for the closest image feature to each template primitive. The distance measure in use is the Euclidean distance from the center of gravity of the image feature to the origin of the primitive's local reference frame (i.e. the location of the primitive's configuration). Although this correspondence method admittedly lacks the robustness required in general inspection applications, and depends upon fairly good image registration, it provides sufficiently accurate results in the case of the inspection task, and has the additional benefit of low computational overhead. In future work the correspondence problem will be considered in more detail. We expect to employ correspondence techniques which are specialized to each geometric primitive type.

Once correspondence has been established, a set of empirical primitives is produced by fitting to the image feature pixel locations. The fitting procedure used is in general determined by the template primitive type. In the current version of the system we use eigenvectors of the feature point scatter matrix to derive the empirical geometric parameters [4] and first threshold out small features. The philosophy in use here is that it is preferable to let a missing feature signal a flaw of omission rather than attempting to interpret an inappropriate geometry. 
(a)

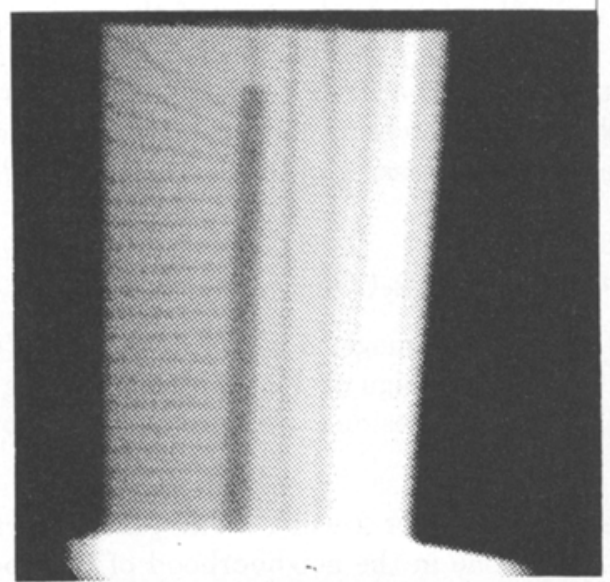

(b)

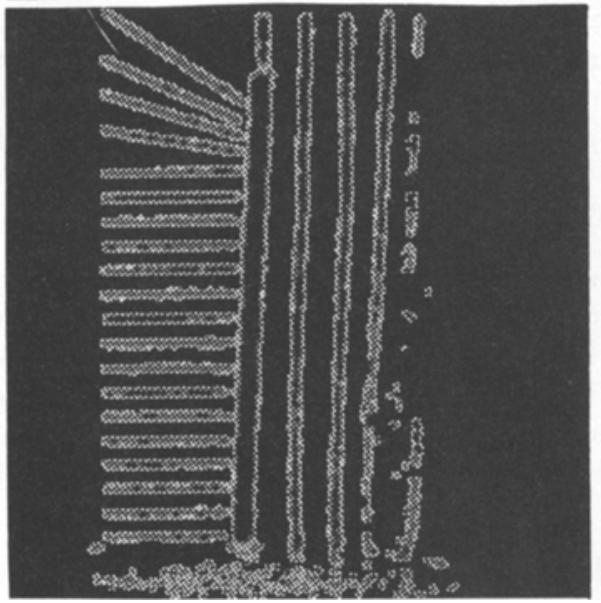

(c)

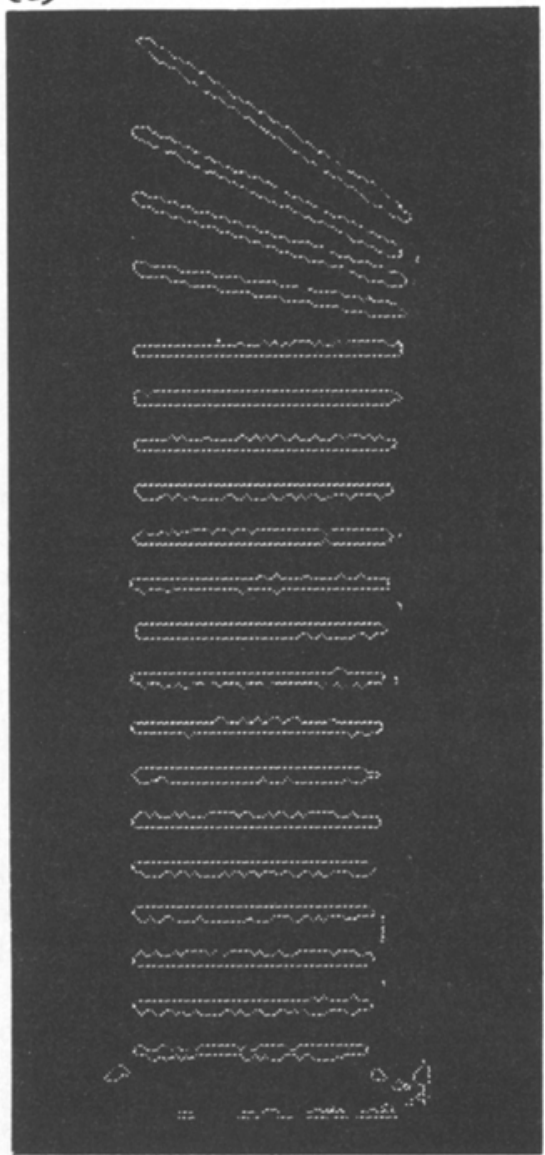

Fig. 3. Drilled hole segmentation: (a) original; (b) region boundary segmentation; (c) subpixel localization of boundaries. In (b) both region edges have been marked which explains the appearance of the boundaries as thick edges.

Constraint Solver. The goal of the constraint solver is to solve the problem of finding an instance of the inspection template which satisfies all of the geometric constraints defined by the template and at the same time, minimizes the mean-square error between the template primitives and the inage features. The mean-square error can be expressed as a convex function of the template parameters and a geometric description of the image features.

Theoretical details of the approach can be found in [12]. Briefly, the two goals of finding the global minimum of a convex function, $\nabla f(\mathbf{x})=0$, and satisfying the constraints, $\mathbf{h}(\mathbf{x})=\mathbf{0}$, are combined to give a constrained minimization problem. A linear approximation to this optimization problem is:

$$
\begin{aligned}
\nabla^{2} f(x) d x & =-\nabla f(x) \\
\nabla \mathbf{h}(\mathbf{x}) d \mathbf{d} & =-\mathbf{h}(\mathbf{x})
\end{aligned}
$$

Since the two goals cannot in general be simultaneously satisfied, a least-square-error satisfaction of $\nabla f(\mathbf{x})=0$ is sought. The constraint equations are multiplied by a factor 
$\sqrt{c}$, which determines the weight given to satisfying the constraints versus minimizing the cost function. Each iteration of (2) has a line search that minimizes the least-square-error:

$$
m(\mathbf{x})=|\nabla f(\mathbf{x})|^{2}+c|\mathbf{h}(\mathbf{x})|^{2}
$$

which is a merit function similar to the objective of the standard penalty method.

Verification. The output from the constraint solver is a set of deformed primitives which can by used for one of two purposes; either to further refine the parameter values and tolerances of the inspection template, or for flaw decision-making. For example, the derived parameters from the deformed primitives can be compared to the template parameters to detect geometric flaws such as inaccurate drilled hole diameters. The deformed inspection template primitives can also provide the context for applying specialized algorithms for characterizing shape and intensity-based properties of subtle flaws. Although the detection of flaws is not the focus of this paper, preliminary results of flaw analysis will be illustrated in the experiments described in the next section.

\section{Experiments}

In this section, we present results from our current working system in action. This system has been designed using object-oriented methodology and implemented on SPARC workstations using the $\mathrm{C}++$ language and the $\mathrm{X}$-based graphics toolkit InterViews.

Template Creation. First, in Figure 2b, we illustrate the process of template creation. The set of configurations in the template contains a number of lines and a number of points. These geometric entities (or rather their counterparts in the inage) are subjected to a number of constraints. The original template and the template after deformation are shown in figure 4 .

(a)
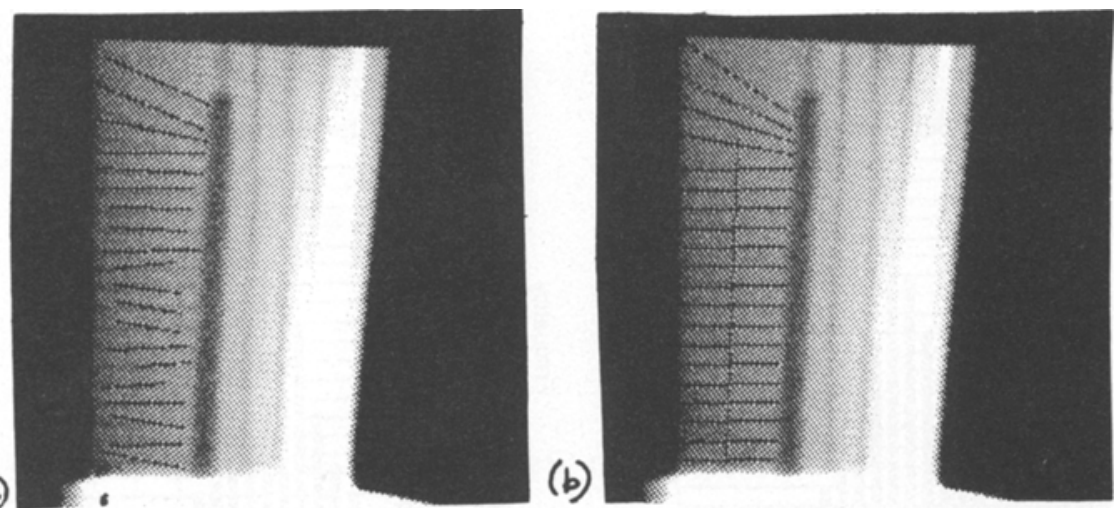

Fig. 4. Template creation and solving for the best fit. (a) Template specification ; (b) Template after best fit.

Feature Tolerance Measurement. Next, we consider using inspection templates to acquire statistical tolerance information. Average values, and variations of length (ie size) and location parameters were collected for the 16 horizontal drill holes of a sample set of 10 "good" parts using the same inspection template. Table 1 shows the results for a selection of holes; numbers $4,6,8$ and 14 from the top. Here the lengths have been normalized by the lengths output from the constraint solver. A histogram plot of the 
Table 1. Normalized nominal values and tolerances for geometric measurements collected over a sample set of 10 images using an inspection template.

\begin{tabular}{|l|r|r|l|l|}
\hline measurement & average & s.d. & max & $\min$ \\
\hline length of hole 4 & 0.997 & 0.011 & 1.020 & 0.974 \\
length of hole 6 & 0.995 & 0.062 & 1.010 & 0.971 \\
length of hole 8 & 0.995 & 0.021 & 1.018 & 0.971 \\
length of hole 14 & 0.998 & 0.011 & 1.018 & 0.984 \\
hole length for sample set & 0.998 & 0.010 & 1.023 & 0.971 \\
outer boundary orientation & 1.017 & 0.16 & 1.220 & 0.807 \\
sum of hole spacing & 0.998 & 0.002 & 1.002 & 0.996 \\
hole separation & 1.000 & 0.018 & 1.132 & 0.956 \\
\hline
\end{tabular}

normalized lengths for the 10 parts is shown in Figure 5a. Table 1 also shows statistics for the sum of hole spacings, hole separation and the orientation of the outer boundary. These global measurements were specified in the template by linear spacing and linear size constraints. As the table shows, the agreement between the template model and image data is very good. This indicates the template accurately represents both critical local and global geometric parameters.

Geometric Flaw Detection. Finally, we illustrate how a template can be used to detect geometric flaws. An image of an industrial part containing a flaw was analyzed by the system using the same inspection template as above. The normalized lengths of the drill holes were recorded as before. Figure 5b shows the updated histogram plot. As seen from the histogram one sample (unshaded) is more than $2 \sigma$ from the sample average. This sample corresponds to the defect drill hole (known as underdrill).

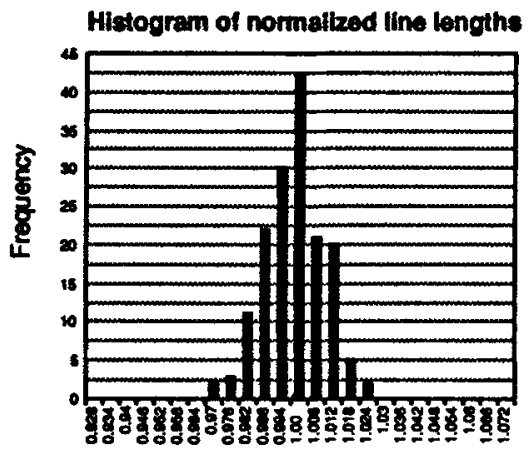

(a)
Normalized length

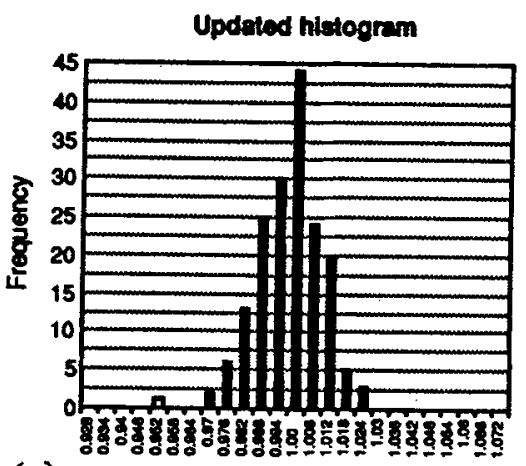

(b)
Normalized length

Fig. 5. Histogram plots of (a) the lengths of holes for a sample set of 10 good parts. Lengths have been normalized by the template values; and, (b) the updated histogram where the samples from a part containing a defect has been added to the sample set. The unshaded sample is more than $2 \sigma$ from the sample average. 


\section{Discussion}

To summarize, this paper has described progress toward the developnent of a geometrybased image analysis system based on the concept of a deformable inspection template. We have described aspects of our approach, some of the key components of our integrated system and presented results from processing experimental data using our current implementation.

Our approach differs from elastic 'snake' based techniques $[1,6]$ and intensity-based deformable parameterized contours [15] and templates [7] in a number of respects. First, we use geometric primitives rather than intensity based features as subcomponents to build the template, although the constraint solving machinery could be modified to handle this case. Second, a key idea our work addresses is how to use a deformable template for quantitative interpretation as opposed to feature extraction. Finally, our scheme allows for the derivation of generic deformable parameterized templates, which is clearly a major benefit for fast prototyping of new inspection algorithms.

\section{References}

1. Burr, D.J.: A Dynamic Model for Image Registration, Computer Vision, Graphics, and Image Processing, 1981, 15,102-112.

2. Chen, C., Mulgaonkar, P.: CAD-Based Feature-Utility Measures For Automatic Vision Programming, Proc. IEEE Workshop Auto. CAD-Based Vision, Lahaina HI, June 1991,106.

3. Farouki, R.: The approximation of non-degenerate offset surfaces, Computer Aided Geometry Design, 1986, 3:1, 15-44.

4. Horn, B.K.P.: Robot Vision, McGraw-Hill, New York, 1986.

5. Huang. G: A robotic alignment and inspection system for semiconductor processing, Int. Conf. on Robot Vision and Sensory Control, Cambridge MA, 1983, 644-652.

6. Kass, M., Witkin, A., Terzopoulos, D.: Snakes: Active Contour Models, Int. J. of Computer Vision, 1988, 1:4, 321-331.

7. Lipson, P., et al.: Deformable Templates for Feature Extraction from Medical Images, Proc. Europ. Conf. on Computer Vision, Antibes France, April 1990, 413-417.

8. Medioni, G., Huertas, A.: Detection of Intensity Changes with Subpixel Accuracy using Laplacian-Gaussian Masks, IEEE PAMI, September 1986, 8:5,651-664.

9. Maragos, P., Schafer, R.W.: Morphological Systems for Multidimensional Signal Processing, Proc. of the IEEE, April 1990, 78:4, 690-710.

10. Nalwa,V.S.: Edge Detector Resolution Improvement by Image Interpolation, IEEE PAMI, May 1987, 9:3, 446-451.

11. Nelson, G.: Juno, a Constraint-Based Graphics System, ACM Computer Graphics, SIGGRAPH '85, San Francisco CA, 1985, 19:3, 235-243.

12. Nguyen, V.,Mundy, J.L., Kapur, D.: Modeling Generic Polyhedral Objects with Constraints, Proc. IEEE Conf. Comput. Vis. \& Patt. Recog., Lahaina HI, June 1991, 479-485.

13. Noble, J.A.: Finding Half Boundaries and Junctions in Images, Accepted Image and Vision Computing, (in press 1992).

14. Okamoto, K., et al.: An automatic visual inspection system for LSI photomasks, Proc. Int. Conf. on Pattern Recognition, Montreal Canada, 1984, 1361-1364.

15. Staib, L.H., Duncan, J.S.: Parametrically Deformable Contour Models, Proc. IEEE Conf. Comput. Vis. \& Patt. Recog., San Diego CA, June 1989, 98-103.

16. West, A., Fernando, T., Dew, P.: CAD-Based Inspection: Using a Vision Cell Demonstrator. Proc. IEEE Workshop on Auto. CAD-Based Vision, Lahaina HI, June 1991, pp 155. 